\title{
Lithium-Ion battery State of Charge estimation with a Kalman Filter based on a electrochemical model
}

\author{
Domenico Di Domenico, Giovanni Fiengo and Anna Stefanopoulou
}

\begin{abstract}
Lithium-ion battery is the core of new plug-in hybrid-electrical vehicles (PHEV) as well as considered in many 2nd generation hybrid electric vehicles (HEV). In most cases the lithium-ion battery performance plays an important role for the energy management of these vehicles as high-rate transient power source cycling around a relatively fixed state of charge (SOC). In this paper an averaged electrochemical Lithium-ion battery model suitable for estimation is presented. The model is based on an averaged approximated relationship between (i) the Butler-Volmer current and the solid concentration at the interface with the electrolyte and (ii) the battery current and voltage. A 4th order model based extended Kalman filter (EKF) is then designed and the estimation results are tested in simulation with the non-averaged model.
\end{abstract}

\section{INTRODUCTION}

Micro-macroscopic battery electrochemical modeling is connected with the hybrid vehicle design, scale-up, optimization and control issues of Hybrid-electrical vehicles (HEV), where the battery plays an important role in this area as high-rate transient power source. When the batteries operate in a relative limited range of state of charge, high efficiency, slow aging and no damaging are expected. As consequence, the state of charge (SOC) estimation and regulation is one of the most important and challenging tasks for hybrid and electrical vehicle control.

Several techniques have been proposed for the SOC estimation, like model based observers or black-box methods (as an example using fuzzy-logic [12]). The accuracy reached by these estimations is about $2 \%$ [9]. Since the more complete models are based on electrochemistry laws [4], [15], [18], a SOC estimation based on these models can improve this precision. The electrochemical models are generally preferred to the equivalent circuit or to other kinds of simplified models, because they also predict the physical cells limitations, which have a relevant effect in the automotive application, where the battery suffers very often the stress of very high transient loads [13].

The importance of Lithium-ion battery has grown in the past few years. Based on the the third lightest element, this kind of battery is widely used in the hybrid vehicles, thanks to its high energy-to-weight ratios, no memory effect and a slow loss of charge when not in use. As the majority of advanced battery systems, Lithium-ion batteries employ porous electrodes in order to increase the active area between

Domenico Di Domenico, Giovanni Fiengo are with Dipartimento di Ingegneria, Università degli Studi del Sannio, Piazza Roma 21, 82100 Benevento. E-mail: \{domenico.didomenico,gifiengo\}@ unisannio.it

Anna Stefanopoulou is with Mechanical Engineering Univ of Michigan, Ann Arbor MI 48109-2121. E-mail: annastef@umich.edu. She is funded by NSF CMS-GOALI 0625610. electrolyte and solid active material, facilitating the electrochemical reactions [2], [10], [13], [14]. Unfortunately, such porosity increases also the model complexity.

Thus, a macroscopic description of the cell is needed. A micro-macroscopic coupled model meeting this requirement, with microscopic and interfacial phenomena, as described in the porous electrode theory, rigorously and systematically integrated into a macroscopic battery model, can be found in [16]. In the porous electrode theory, developed by Newman and Tiedemann [7], the electrode is treated as a superposition of two continua, namely the electrolytic solution and the solid matrix. The solid matrix is modeled as a microscopic sphere, where the solid active material diffuses and reacts on the spheres surface, as shown in Figure 1.

These models predict the solid concentration profile during charge and discharge, but, unfortunately, a single point solid concentration estimation along the electrodes is difficult to realize with a real-time on-board estimator, due to the complexity of the model. As a consequence several approximation are typically introduced between the input and output of the dynamical system and the battery input and output to reduce the system order and complexity [2], [8].

In this paper we employ an approximation and present an extended Kalman filter for SOC estimation. In the following, a general micro-macroscopic Lithium-ion battery model, as it is presented in literature, is summarized. Then the model is simplified in order to make it compatible with a feasible solid concentration estimation. Finally an Extended Kalman Filter (EKF) is designed based on the averaged model. The estimation results are compared with the full micromacroscopic model prediction.

\section{BATTERY GENERAL FEATURE}

A battery is composed of three parts: the two electrodes and the separator. Referring to a porous battery, each electrode consists of a solid matrix inside an electrolyte solution, while the separator is just made from the electrolyte solution. In particular, for a Lithium-ion battery, the negative electrode, or anode, is composed of carbon, the positive electrode, or cathode, is a metal oxide and the electrolyte is a lithium salt in an organic solvent, such as $\mathrm{LiPF}_{6}, \mathrm{LiBF}_{4}$ or $\mathrm{LiClO}_{4}$.

The separator is a solid or liquid solution with high concentration of lithium ion. It conducts the ion but it is an electronic insulator. At the negative electrode, the solid active material particles of lithium $\left(\mathrm{Li}_{x} \mathrm{C}_{6}\right)$ diffuse to the electrolyte-solid interface where the chemical reaction occurs, transferring the lithium ions to the solution and 


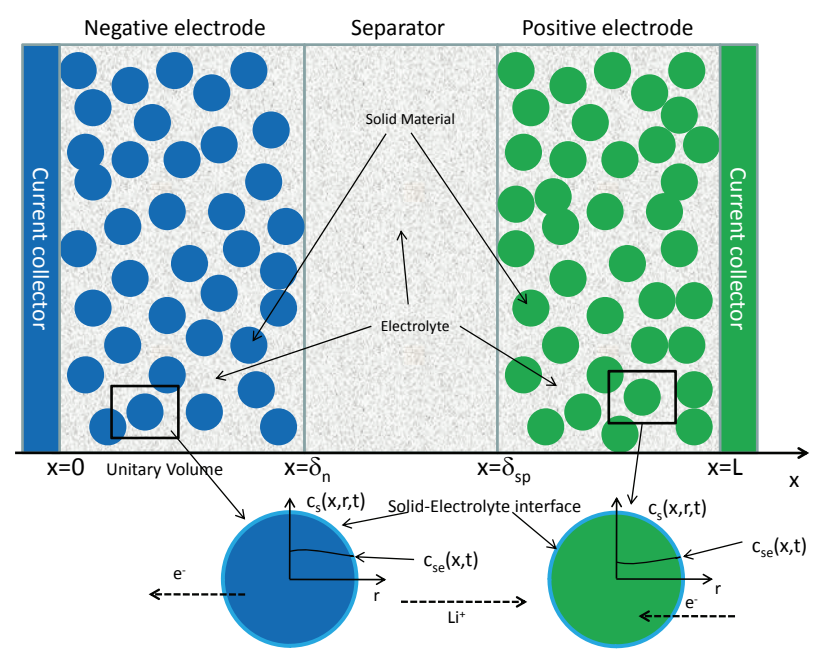

Fig. 1. Schematic macroscopic ( $x$-direction) cell model with coupled microscopic ( $r$-direction) solid diffusion model.

the electrons to the collector [13]. The produced electrolyte material goes through the solution to the positive electrode, where, at the interface with solid material, it reacts and inserts into the metal oxide solid particles.

It's generally accepted that a microscopic description of the battery is intractable, due to the complexity of the interfaces [16]. So, in order to mathematically model the battery, both macroscopic and microscopic physics have to be considered.

The resulting equations describe the battery system with four quantities, i.e. solid and electrolyte concentrations $\left(c_{s}\right.$, $\left.c_{e}\right)$ and solid and electrolyte potentials $\left(\phi_{s}, \phi_{e}\right)$. The complete set of equations describing the micro-macroscopic model is [5], [13]

$$
\begin{gathered}
i_{e}(x)=-\kappa^{e f f} \vec{\nabla}_{x} \phi_{e}-\kappa_{D}^{e f f} \vec{\nabla}_{x} \ln c_{e} \\
i_{s}(x)=-\sigma^{e f f} \vec{\nabla}_{x} \phi_{s} \\
\vec{\nabla}_{x} i_{e}(x)=j^{L i} \\
\vec{\nabla}_{x} i_{s}(x)=-j^{L i} \\
\frac{\partial \epsilon_{e} c_{e}}{\partial t}=\vec{\nabla}_{x}\left(D_{e}^{e f f} \vec{\nabla}_{x} c_{e}\right)+\frac{1-t^{0}}{F} j^{L i} \\
\frac{\partial c_{s}}{\partial t}=\vec{\nabla}_{r}\left(D_{s} \vec{\nabla} c_{s}\right)
\end{gathered}
$$

with the Butler-Volmer current density

$$
j^{L i}(x)=a_{s} j_{0}\left[\exp \left(\frac{\alpha_{a} F}{R T} \eta\right)-\exp \left(-\frac{\alpha_{c} F}{R T} \eta\right)\right]
$$

where the overpotential $\eta$ is obtained as

$$
\eta=\phi_{s}-\phi_{e}-U\left(c_{s e}\right)
$$

and $U$ is the open circuit voltage, function of the solid concentration at the electrolyte interface indicated with $c_{s e}(x, t)=c_{s}\left(x, R_{s}, t\right)$. The variables $R$ and $F$ are the universal gas and the Faraday's constants and $T$ is the absolute temperature. The open circuit voltage for the negative electrode, denoted with the subscript $n$, is calculated using the empirical correlation introduced in [3]

$$
\begin{aligned}
& U_{n}\left(\theta_{n}\right)=8.0029+5.0647 \theta_{n}-12.578 \theta_{n}^{0.5} \\
& -8.6322 \times 10^{-4} \theta_{n}^{-1}+2.1765 \times 10^{-5} \theta_{n}^{3 / 2} \\
& -0.46016 \exp \left[15.0\left(0.06-\theta_{n}\right)\right] \\
& -0.55364 \exp \left[-2.4326\left(\theta_{n}-0.92\right)\right]
\end{aligned}
$$

where $\theta_{n}(x)=c_{s e} / c_{s, \text { max }}^{a n o d e}$ is the normalized solid concentration at the anode. For the positive electrode, denoted with $p$, the result obtained from [13] has been adopted

$$
\begin{aligned}
& U_{p}\left(\theta_{p}\right)=85.681 \theta_{p}^{6}-357.70 \theta_{p}^{5}+613.89 \theta_{p}^{4} \\
& -555.65 \theta_{p}^{3}+281.06 \theta_{p}^{2}-76.648 \theta_{p} \\
& +13.1983-0.30987 \exp \left(5.657 \theta_{p}^{115}\right)
\end{aligned}
$$

where $\theta_{p}(x)=c_{s e} / c_{s, \text { max }}^{\text {cathode }}$ is the normalized solid concentration at the cathode. The coefficient $j_{0}$ in (7) also exhibits a modest dependence on the solid and electrolyte concentration, according to

$$
j_{0}=\left(c_{e}\right)^{\alpha_{a}}\left(c_{s, \max }-c_{s e}\right)^{\alpha_{a}}\left(c_{s e}\right)^{\alpha_{c}} .
$$

Finally, the cell potential which is typically measured is computed as

$$
V=\phi_{s}(x=L)-\phi_{s}(x=0)-R_{f} I
$$

where $R_{f}$ is the film resistance on the electrodes surface. In Figure 2 the model equations and their boundary conditions for the $x$-domain and $r$-domain are shown. Note that the temperature spatial and temporal gradients are neglected in this work. For completeness the battery model parameters are summarized in Table I and more details on the model and its parameters can be found in [13], [17].

\section{REDUCED ORDER MODEL}

For a state space formulation, let the battery current $I$ be the model input which governs the boundary conditions of (1)-(6) as shown in Fig. 2. For each $x$-dimension discretization step, an ODE is obtained. This results in $2 N_{x}$ different systems $\left(N_{x}\right.$ for the anode and $N_{x}$ for the cathode), each of $\left(M_{r}-1\right)$-order, driven by a time-dependent nonlinear function of the battery input $I$ through the ButlerVolmer current. The discretized PDE system of (6) at the $l$ location along the $\mathrm{x}$-dimension forms the $l$-state $\mathbf{c}_{\mathbf{s}}=$ $\left(c_{s_{1}}, c_{s_{2}}, \ldots . c_{s_{M_{r}-1}}\right)^{T}$ showing explicitly the dependency on the Butler-Volmer current $j_{l}^{L i}$

$$
\left\{\begin{array}{l}
\dot{c}_{s_{q}}=\left(\frac{q+1}{q}\right) \alpha_{1} c_{s_{q+1}}-2 \alpha_{1} c_{s_{q}}+\left(\frac{q-1}{q}\right) \alpha_{1} c_{s_{q-1}} \\
\dot{c}_{s_{M_{r}-1}}=\left(\frac{M_{r}-2}{M_{r-1}}\right) \alpha_{1} c_{s_{M_{r}-2}}-\left(\frac{M_{r}-2}{M_{r}-1}\right) \alpha_{1} c_{s_{M_{r}-1}} \\
-\left(\frac{M_{r}}{M_{r}-1}\right) \alpha_{2} j_{l}^{L i}
\end{array}\right.
$$

with $q=1, \ldots, M_{r}-2, l=1, \ldots, N_{x}, \alpha_{1}=D_{s} / \Delta_{r}^{2}$ and $\alpha_{2}=\left(F a_{s} / \Delta_{r}\right)^{-1}$ and $j_{l}^{L i}\left(\mathbf{c}_{\mathbf{s}}, I\right)=\mathcal{U}_{l}\left(\mathbf{c}_{\mathbf{s}}, I\right)$, where 
TABLE I

BATTERY PARAMETERS.

\begin{tabular}{|c|c|c|c|}
\hline Parameter & Negative electrode & Separator & Positive electrode \\
\hline Thickness $(\mathrm{cm})$ & $\delta_{n}=50 \times 10^{-4}$ & $\overline{\delta_{\text {sep }}=25.4 \times 10^{-4}}$ & $\overline{\delta_{p}}=36.4 \times 10^{-4}$ \\
\hline Particle radius $R_{s}(\mathrm{~cm})$ & $1 \times 10^{-4}$ & - & $1 \times 10^{-4}$ \\
\hline Active material volume fraction $\varepsilon_{s}$ & 0.580 & - & 0.500 \\
\hline Electrolyte phase volume fraction (porosity) $\varepsilon_{e}$ & 0.332 & 0.5 & 0.330 \\
\hline $\begin{array}{l}\text { Conductivity of solid active material } \\
\sigma\left(\Omega^{-1} \mathrm{~cm}^{-1}\right)\end{array}$ & 1 & - & 0.1 \\
\hline Effective conductivity of solid active material & $\sigma^{e f f}=\varepsilon_{s} \sigma$ & - & $\sigma^{e f f}=\varepsilon_{s} \sigma$ \\
\hline Transference number $t_{+}^{0}$ & 0.363 & 0.363 & 0.363 \\
\hline $\begin{array}{l}\text { Electrolyte phase ionic conductivity } \\
\kappa\left(\Omega^{-1} \mathrm{~cm}^{-1}\right)\end{array}$ & $\kappa=0.0158 c_{e} \exp \left(0.85 c_{e}^{1.4}\right)$ & $\kappa=0.0158 c_{e} \exp \left(0.85 c_{e}^{1.4}\right)$ & $\kappa=0.0158 c_{e} \exp \left(0.85 c_{e}^{1.4}\right)$ \\
\hline Effective electrolyte phase ionic conductivity & $\kappa^{e f f}=\left(\varepsilon_{e}\right)^{1.5} \kappa$ & $\kappa^{e f f}=\left(\varepsilon_{e}\right)^{1.5} \kappa$ & $\kappa^{e f f}=\left(\varepsilon_{e}\right)^{1.5} \kappa$ \\
\hline Effective electrolyte phase diffusional conductivity & $\stackrel{\kappa_{D}^{e f f}}{\kappa^{\prime}}=\frac{2 R T \kappa^{e f f}}{F}\left(t_{+}^{0}-1\right)$ & $\kappa_{D}^{e f f}=\frac{2 R T \kappa^{e f f}}{F}\left(t_{+}^{0}-1\right)$ & $\kappa_{D}^{e f f}=\frac{2 R T \kappa^{e f f}}{F}\left(t_{+}^{0}-1\right)$ \\
\hline $\begin{array}{l}\text { Electrolyte phase diffusion coefficient } \\
D_{e}\left(\mathrm{~cm}^{2} \mathrm{~s}^{-1}\right)\end{array}$ & $2.6 \times 10^{-6}$ & $2.6 \times 10^{-6}$ & $2.6 \times 10^{-6}$ \\
\hline Effective electrolyte phase diffusion coefficient & $D_{e}^{e f f}=\left(\varepsilon_{e}\right)^{1.5} D_{e}$ & $D_{e}^{e f f}=\left(\varepsilon_{e}\right)^{1.5} D_{e}$ & $D_{e}^{e f f}=\left(\varepsilon_{e}\right)^{1.5} D_{e}$ \\
\hline Solid phase diffusion coefficient $D_{s}\left(\mathrm{~cm}^{2} \mathrm{~s}^{-1}\right)$ & $2.0 \times 10^{-12}$ & - & $3.7 \times 10^{-12}$ \\
\hline $\begin{array}{l}\text { Maximum solid-phase concentration } \\
c_{s, \max }\left(\mathrm{mol} \mathrm{cm}^{-3}\right)\end{array}$ & $16.1 \times 10^{-3}$ & - & $23.9 \times 10^{-3}$ \\
\hline Average electrolyte concentration $\bar{c}_{e}\left(\mathrm{~mol} \mathrm{~cm} \mathrm{~cm}^{-3}\right)$ & $1.2 \times 10^{-3}$ & $1.2 \times 10^{-3}$ & $1.2 \times 10^{-3}$ \\
\hline Change transfers coefficients $\alpha_{a}, \alpha_{c}$ & $0.5,0.5$ & - & $0.5,0.5$ \\
\hline $\begin{array}{l}\text { Active surface area per electrode unit volume } \\
a_{s}\left(\mathrm{~cm}^{-1}\right)\end{array}$ & $a_{s_{n}}=\frac{3 \varepsilon_{e}}{R_{s}}$ & - & $a_{s_{p}}=\frac{3 \varepsilon_{e}}{R_{s}}$ \\
\hline Electrode plate area, $A\left(\mathrm{~cm}^{2}\right)$ & 10452 & - & 10452 \\
\hline Film resistance at electrode surface, $R_{f}(m \Omega)$ & 20 & - & 20 \\
\hline
\end{tabular}

the dependence on the input of the Butler-Volmer current is explicitly highlighted. It follows

$$
\dot{\mathbf{c}}_{\mathbf{s}}=A \mathbf{c}_{\mathbf{s}}+b \mathcal{U}_{l}\left(\mathbf{c}_{\mathbf{s}}, I\right)
$$

where $A$ and $b$ can be determined from (13) and the $\mathcal{U}_{l}$ can be considered as a set of $N_{x}$ parameters, each of them appearing in one of the respective $N_{x}$ state-space systems, and has to be derived from (1)-(6). The output of the system is the value of the solid concentration on the sphere radius, that can be rewritten

$$
c_{s e}=c_{s_{M-1}}-\frac{\alpha_{2}}{\alpha_{1}} \mathcal{U}_{l}\left(\mathbf{c}_{\mathbf{s}}, I\right) .
$$

Note that the positive and negative electrode dynamical systems differ for the constant values and for the input expression through equations (7)-(10). Furthermore, along each electrode, as the boundary condition, i.e. the input $\mathcal{U}_{l}$, depends on $x$ the system output in also function of $x$.

A model simplification can be achieved by neglecting the solid concentration distribution along the electrode and considering the material diffusion inside a representative solid material particle for each electrode. That introduces an average value of the solid concentration that can be related with the definition of battery state of charge. Although this simplified model results in a heavy loss of information, it can be useful in control and estimation applications. In accordance with the mean solid concentration, the spacial dependence of the Butler-Volmer current is ignoredand a constant value $\bar{j}^{L i}$ is considered which satisfy the spacial integral of (3) or (4), giving for the anode (and can be reproduced accordingly in the cathode)

$$
\int_{0}^{\delta_{n}} j^{L i}\left(x^{\prime}\right) d x^{\prime}=\frac{I}{A}=\bar{j}_{n}^{L i} \delta_{n}
$$

where $\delta_{n}$ is the anode thickness, as shown in Figure 1. The battery voltage (12) using (8) can be rewritten as

$$
\begin{aligned}
V(t)= & \eta(L, t)-\eta(0, t)+\left(\phi_{e}(L, t)-\phi_{e}(0, t)\right) \\
& +\left(U_{p}\left(c_{s e}(L, t)\right)-U_{n}\left(c_{s e}(0, t)\right)\right)-R_{f} I
\end{aligned}
$$

and using the average values at the anode and the cathode instead of the boundary values the following relation is obtained

$$
\begin{array}{r}
V(t)=\bar{\eta}_{p}-\bar{\eta}_{n}+\left(\bar{\phi}_{e, p}-\bar{\phi}_{e, n}\right) \\
+\left(U_{p}\left(\bar{c}_{s e, p}\right)-U_{n}\left(\bar{c}_{s e, n}\right)\right)-R_{f} I .
\end{array}
$$

Using the microscopic current average values and imposing the boundary conditions and the continuity at the interfaces, the solutions of equations (1) - (6) are, for the anode

$$
\begin{array}{r}
\phi_{e}(x)=\phi_{e}(0)-\frac{I}{2 A k^{e f f}} \delta_{n} x^{2} \\
\phi_{s}(x)=-\frac{I}{A \sigma^{e f f}}\left(x-\frac{1}{2 \delta_{n} x^{2}}\right)
\end{array}
$$

for the separator

$$
\phi_{e}(x)=\phi_{e}(0)-\frac{I}{2 A k^{e f f}} \delta_{n}-\frac{I}{A k^{e f f}}\left(x-\delta_{n}\right)
$$

and for the cathode

$$
\begin{array}{r}
\phi_{e}(x)=\phi_{e}(0)-\frac{I}{2 A k^{e f f}} \delta_{n}-\frac{I}{A k^{e f f}} \delta_{s e p}+ \\
\frac{I}{2 A k^{e f f} \delta_{p}}\left(x-\delta_{s p}\right)^{2}-\frac{I}{A k^{e f f}}\left(x-\delta_{s p}\right)
\end{array}
$$




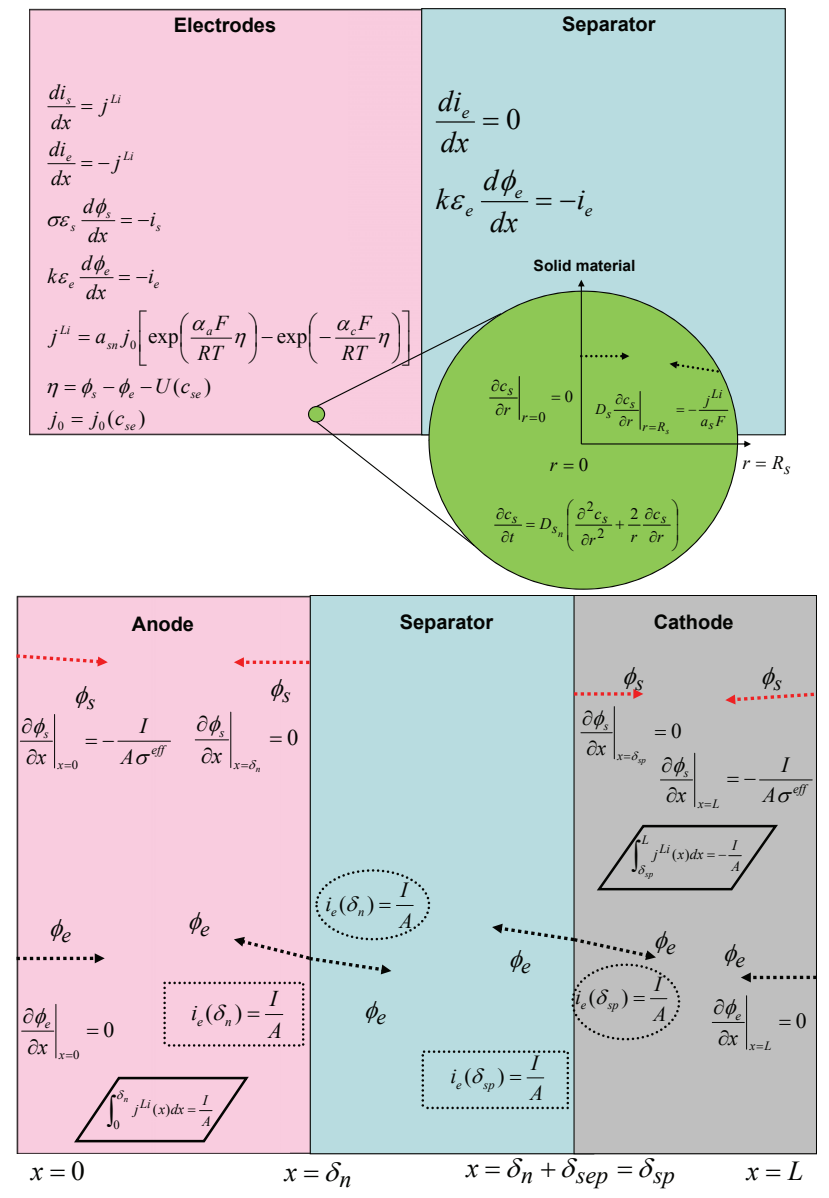

Fig. 2. Schematic representation of the set of equations and of the boundary conditions for the potentials and solid concentration. The current I is assumed to be positive (battery discharge) and provide boundary condition (boxes with dotted line) and the non-local constraints (boxes with solid line) governing the Butler-Volmer current density.

$$
\phi_{s}(x)=\phi_{s}(L)-\frac{I}{A \sigma^{e f f}}\left(\left(x-\delta_{s p}\right)-\frac{1}{2 \delta_{n}}\left(x-\delta_{s p}\right)^{2}\right)
$$

where $\delta_{\text {sep }}$ is the separator thickness and $\delta_{s p}=\delta_{n}+\delta_{\text {sep }}$. The approximate solutions (19)-(22) lead to

$\bar{\phi}_{e, p}-\bar{\phi}_{e, n}=\phi_{e}(L)-\phi_{e}(0)=-\frac{I}{2 A k^{e f f}}\left(\delta_{n}+2 \delta_{s e p}+\delta_{p}\right)$.

Furthermore, considering

$$
\begin{gathered}
\left.\bar{j}_{n}^{L i}=\frac{I}{A \delta_{n}}=a_{s} j_{0}\left[\exp \left(\frac{\alpha_{a} F}{R T} \bar{\eta}_{n}\right)\right)-\exp \left(-\frac{\alpha_{c} F}{R T} \bar{\eta}_{n}\right)\right] \\
\left.\bar{j}_{p}^{L i}=-\frac{I}{A \delta_{p}}=a_{s} j_{0}\left[\exp \left(\frac{\alpha_{a} F}{R T} \bar{\eta}_{p}\right)\right)-\exp \left(-\frac{\alpha_{c} F}{R T} \bar{\eta}_{p}\right)\right]
\end{gathered}
$$

$\bar{\eta}_{n}$ and $\bar{\eta}_{p}$ can be estimated as

$$
\begin{aligned}
& \bar{\eta}_{n}=\frac{R T}{\alpha_{a} F} \ln \left(\xi_{n}+\sqrt{\xi_{n}^{2}+1}\right) \\
& \bar{\eta}_{p}=\frac{R T}{\alpha_{a} F} \ln \left(\xi_{p}+\sqrt{\xi_{p}^{2}+1}\right)
\end{aligned}
$$

where

$$
\xi_{n}=\frac{\bar{j}_{n}^{L i}}{2 a_{s} j_{0}} \text { and } \xi_{p}=\frac{\bar{j}_{p}^{L i}}{2 a_{s} j_{0}} .
$$

Finally, the battery voltage (18) can be written as a function of battery current and of the average solid concentration

$$
\begin{aligned}
& V(t)=\frac{R T}{\alpha_{a} F} \ln \frac{\xi_{n}+\sqrt{\xi_{n}^{2}+1}}{\xi_{p}+\sqrt{\xi_{p}^{2}+1}}+\bar{\phi}_{e, p}-\bar{\phi}_{e, n} \\
& +\left(U_{p}\left(\bar{c}_{s e, p}\right)-U_{n}\left(\bar{c}_{s e, n}\right)\right)-R_{f} I .
\end{aligned}
$$
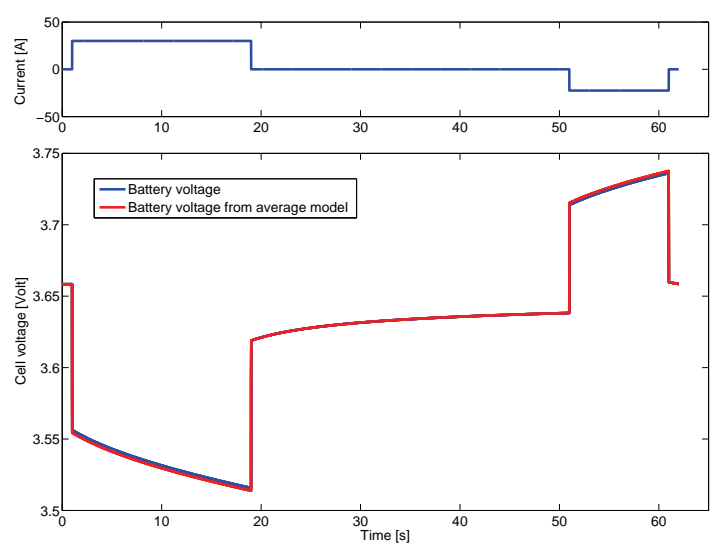

Fig. 3. Average versus complete battery model: output voltage.
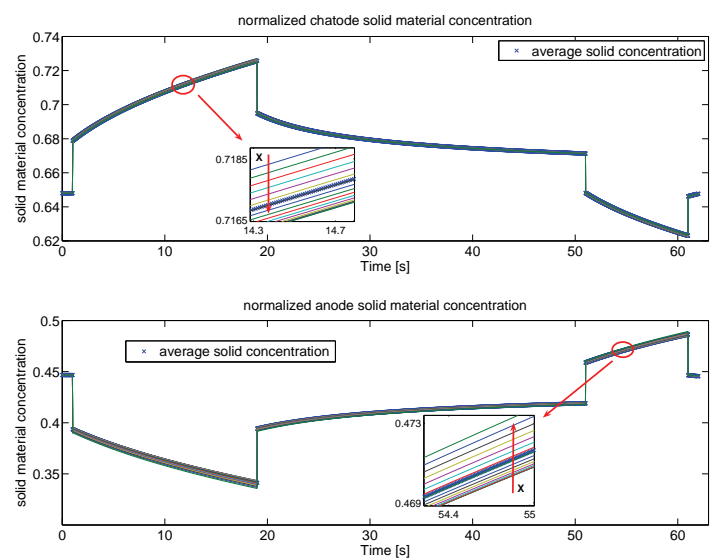

Fig. 4. Average versus complete battery model: anode and cathode solid material concentration. The different lines represent the concentration values along the $\mathrm{x}$-direction.

Figures 3 and 4 demonstrate the performance of the reduced order model driven by $\left(M_{r}-1\right)$-order (14) ordinary equation, comparing the simulated signal with the whole model given by (14) resolved at $N_{x}$ locations across the $x$-direction during a FreedomCAR test procedure, an U.S. Department of Energy program for the zero-emission vehicle and technology research [1]. The test procedures, shown in the top plot of Figure 3, consists of a 30 A battery discharge for $18 \mathrm{~s}$, open-circuit relaxation for $32 \mathrm{~s}, 22.5 \mathrm{~A}$ charge for 
$10 \mathrm{~s}$ followed by open-circuit relaxation. In particular, Figure 3 shows a good battery voltage prediction, with a maximum error of $2 \mathrm{mV}$, while Figure 4 highlights a good agreement between the distributed value of the solid concentration and the predicted average. It's important to note that the averaging procedure for the Butler-Volmer current introduced here, is equivalent to considering a representative solid material particle somewhere along the anode and the cathode.

\section{KALMAN FILTER StATE OF CHARgE ESTIMATION}

In most cases the battery voltage $\mathrm{V}$ is measured, and along the known input (current demanded) one needs to estimate the battery SOC. The physical quantity related to the battery state of charge is the solid concentration at the electrodes. In order to design a Kalman Filter for the on-line SOC estimation based on the electrochemical proposed model, a preliminary observability discussion is required.

Specifically, the average dynamical system describes the diffusion effects into two solid material particles, one for the cathode and one for the anode, and allows to compute the solid concentration at the spheres radius, which represent an average value of the solid concentration along the electrodes. However the cell voltage (33) depends on $\left(U_{p}\left(\bar{c}_{s e, p}\right)-U_{n}\left(\bar{c}_{s e, n}\right)\right)$ making the difference of the open circuit voltage observable but not necessarily each open circuit voltage. Indeed, the system that includes both positive and negative electrode concentration states is weakly observable (in the linear sense) from the output cell voltage.

It's possible to find a relation between the anode and the cathode average solid concentrations which can be used for the estimation of the negative electrode concentration based on the positive electrode. As it is shown below, the positive electrode concentration states are observable from the output cell voltage. Then the voltage measurement is used as an output injection to the positive (alone) electrode concentration observer. This relation can be found starting from the electrodes capacity and the SOC by introducing the stoichiometry ratio $\theta=\frac{\bar{c}_{s e}}{c_{s, \max }}$. Its reference value, $\theta_{100 \%}$, can be defined for each electrode finding the average concentration corresponding to a full charge battery, i.e. $100 \%$ of the state of charge. Thus, the $0 \%$ reference stoichiometry can be derived from $\theta_{100 \%}$ by subtracting the battery capacity $Q$, with the appropriate conversion:

$$
\theta_{0 \%}=\theta_{100 \%}-\frac{Q}{\delta}\left(\frac{1}{A F \varepsilon c_{s, \max }}\right)
$$

where $\delta, \varepsilon$ and $c_{s, \max }$ have appropriate values for anode and cathode. Then, the state of charge of the battery is, with a good approximation, linearly varying with $\theta$ between the two reference values at $0 \%$ and $100 \%$

$$
S O C(t)=\frac{\theta-\theta_{0 \%}}{\theta_{100 \%}-\theta_{0 \%}} .
$$

Equation (30) allows the SOC estimation using a single electrode solid concentration. Because the measured battery voltage depends on both concentrations, the SOC estimation has to account for both electrodes. The negative electrode concentration can be computed using (30) as

$$
\begin{aligned}
& \bar{c}_{s e, n}=c_{s, \max , n} \\
& \left(\theta_{n 0 \%}+\frac{\bar{c}_{s e, p}-\theta_{p 0 \%} c_{s, \max , p}}{\left(\theta_{p 100 \%}-\theta_{p 0 \%}\right) c_{s, \max , p}}\left(\theta_{n 100 \%}-\theta_{n 0 \%}\right)\right)
\end{aligned}
$$

where $\theta_{n 0 \%}, \theta_{n 100 \%}, \theta_{p 0 \%}$ and $\theta_{p 100 \%}$ are the reference stoichiometry points for the anode and the cathode.

Hence, introducing the state vector $x=$ $\left(\bar{c}_{s, p 1}, \bar{c}_{s, p 2}, \ldots . ., \bar{c}_{s, p\left(M_{r}-1\right)}\right)^{T}, \quad$ the dynamical system is

$$
\dot{x}=A_{p} x(t)+B_{p} u(t) \text { with } u=\bar{j}_{p}^{L i} \text { and } y=V(x, u)
$$

where the matrices $A_{p}$ and $B_{p}$ are obtained from (13) with reference to the positive electrode. For a linear state-space formulation, the linearized battery voltage results in an output matrix $C=\partial V / \partial x$ which is a row matrix with zeros in its first $M_{r}-2$ elements and the last non-zero term being

$$
\frac{\partial V}{\partial \bar{c}_{s, p\left(M_{r}-1\right)}}=\frac{\partial U_{p}}{\partial \bar{c}_{s, p\left(M_{r}-1\right)}}-\frac{\partial U_{n}}{\partial \bar{c}_{s e, n}} \frac{\partial \bar{c}_{s e, n}}{\partial \bar{c}_{s, p\left(M_{r}-1\right)}}
$$

due to the fact that the battery potential $V$ is only a function of the solid concentration at interface. This output matrix $C$ leads to a strongly observable system (32).

The non linear system observability was also studied. The (32) leads to a $\left(M_{r}-1\right)$-dimensional codistribution $\mathcal{H}$ of the observation space $H$, which imply that the system is strongly locally observable $\forall \bar{c}_{\text {se,p }} \neq 0$ [6], [11].

Based on the average model developed in the previous section, a Kalman filter can be designed, according to

$$
\begin{aligned}
& \dot{\hat{x}}=A_{p} \hat{x}+B u+K_{e}(y-\hat{y}) \\
& \hat{y}=V(\hat{x}, u)
\end{aligned}
$$

where $\hat{x}$ and $\hat{y}$ are respectively the estimate state and output, $V$ is the output nonlinear function in (28), $A_{p}, B_{p}$ are the matrices describing the dynamical system defined in (32), $C$ defined in (33) and $K_{e}$ is the Kalman gain, obtained as follows

$$
K_{e}=P C R^{-1}
$$

where $P$ is the solution of the Riccati equation

$$
\begin{aligned}
& \dot{P}=A_{p} P+P A_{p}^{T}-P C R^{-1} C^{T} P+Q \\
& P(0)=P_{0},
\end{aligned}
$$

and $Q$ and $R$ are weight matrices appropriately tuned in order to minimize the quadratic error on battery voltage. A Matlab optimization procedure returned $Q=10 \times I$ (where $I$ the identity matrix) and $R=12$.

Figure 5 highlights the filter performance. The 4th order Kalman filter estimation results are compared with the full 300 th order model. The error in the initial condition, close to $10 \%$, is fully and quickly recovered, showing that the filter is able to estimate the correct value of the battery state of charge even if its open loop model prediction was $10 \%$ wrong. The step in current demand results in a step in solid 


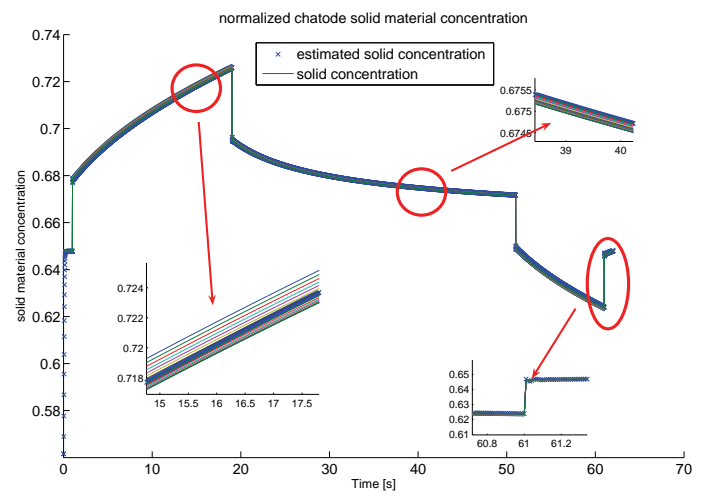

Fig. 5. Kalman Filter: solid concentration estimation.
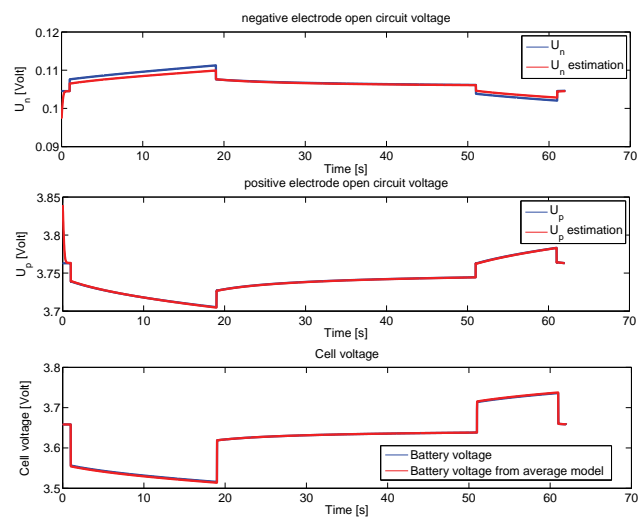

Fig. 6. Kalman Filter: open circuit voltage estimation.

concentration which is again estimated by the filter, as shown in a zoom of the same figure.

Furthermore, the reduced order model based Kalman filter provides also a good estimation for the single electrode open circuit voltage even though just the open circuit voltage $U_{p}-$ $U_{n}$ is observable. It is because the measured output $V$ is not very sensitive to $U_{n}$, so the correct value of the battery voltage can be predicted uniquely by the positive electrode solid concentration, as confirmed by the results shown in Figure 6.

\section{CONCLUSION}

An isothermal electrochemical model of the Lithium-ion battery was used to derive an averaged model coupling the average microscopic solid material concentration with the average values of the chemical potentials, electrolyte concentration and microscopic current density. Finally, an EKF, based on the average model, was designed for the SOC estimation. Its performance was analyzed and the excellent estimation results were shown and discussed.

\section{REFERENCES}

[1] Freedomcar battery test manual for power-assist hybrid elecric veicles. DOE/ID-11069, 2003.

[2] O. Barbarisi, F. Vasca, and L. Glielmo. State of charge kalman filter estimator for automotive batteries. Control Engineering Practice, $14: 267-275,2006$
TABLE II

LITHIUM-ION MODEL NOMENCLATURE.

\begin{tabular}{|c|c|c|}
\hline Symbol & Name & Unit \\
\hline \hline$i_{e}$ & electrolyte current density & $\mathrm{A} \mathrm{cm}^{-2}$ \\
\hline$i_{s}$ & solid current density & $\mathrm{A} \mathrm{cm}^{-2}$ \\
\hline$\phi_{e}$ & electrolyte potential & $\mathrm{V}$ \\
\hline$\phi_{s}$ & solid potential & $\mathrm{V}$ \\
\hline$c_{e}$ & electrolyte concentration & $\mathrm{mol} \mathrm{cm}^{-3}$ \\
\hline$c_{s}$ & solid concentration & $\mathrm{mol} \mathrm{cm}^{-3}$ \\
\hline$c_{s e}$ & solid concentration at electrolyte interface & $\mathrm{mol} \mathrm{cm}^{-3}$ \\
\hline$j^{L i}$ & microscopic Butler-Volmer current & $\mathrm{A} \mathrm{cm}^{-3}$ \\
\hline$\theta_{n}$ & normalized solid concentration at anode & \\
\hline$\theta_{p}$ & normalized solid concentration at cathode & \\
\hline $\mathrm{U}$ & open circuit voltage & $\mathrm{V}$ \\
\hline$U_{n}$ & anode open circuit voltage & $\mathrm{mol} \mathrm{cm}^{-3}$ \\
\hline$U_{p}$ & cathode open circuit voltage & $\mathrm{mol} \mathrm{cm}$ \\
\hline$\eta$ & overpotential & $\mathrm{V}$ \\
\hline $\mathrm{F}$ & Faraday's number & $\mathrm{C}$ \\
\hline $\mathrm{I}$ & battery current & $\mathrm{A}$ \\
\hline $\mathrm{R}$ & gas constant & $\mathrm{V}$ \\
\hline $\mathrm{T}$ & temperature & $\mathrm{V}$ \\
\hline $\mathrm{V}$ & voltage & $\mathrm{C}$ \\
\hline $\mathrm{Q}$ & capacity & \\
\hline & & \\
\hline
\end{tabular}

[3] M. Doyle and Y. Fuentes. Computer simulations of a lithium-ion polymer battery and implications for higher capacity next-generation battery designs. J. Electrochem. Soc., 150:A706-A713, 2003.

[4] M. Doyle, T.F. Fuller, and J. Newman. Modeling of galvanostatic charge and discharge of the lithium/polymer/insertion cell. J. Electrochem. Soc., 140, 1993.

[5] W.B. Gu and C.Y. Wang. Thermal and electrochemical coupled modeling of a lithium-ion cell. Proceedings of the ECS, 99, 2000.

[6] R. Hermann and A. J. Krener. Nonlinear controllability and observability. IEEE Trans. Aut. Contr., pages 728-740, 1977.

[7] J. Newman and W. Tiedemann. Porus-electrode theory with battery applications. AIChE Jurnal, 21, 1975.

[8] B. Paxton and J. Newmann. Modeling of nickel metal hydride. Journal of Electrochemical Society, 144, 1997

[9] V. Pop, H. J. Bergveld, J. H. G. Op het Veld, P. P. L. Regtien, D. Danilov, and P. H. L. Notten. Modeling battery behavior for accurate state-of-charge indication. J. Electrochem. Soc., 153, 2006.

[10] J.A. Prins-Jansen, J.D. Fehribach, K. Hemmes, and J.H.W. de Wita. A three-phase homogeneous model for porous electrodes in moltencarbonate fuel cells. J. Electrochem. Soc., 143, 1996.

[11] W. Respondek. Geometry of Static and Dynamic Feedback. in Mathematical Control Theory, Lectures given at the Summer Schools on Mathematical Control Theory Trieste, Italy, 2001.

[12] A.J. Salkind, C. Fennie, P. Singh, T. Atwater, and D.E. Reisner. Determination of state-of-charge and state-of-health of batts. by fuzzy logic methodology. J. Power Sources, 80:293-300, 1999.

[13] K. Smith and C.Y. Wang. Solid-state diffusion limitations on pulse operation of a lithium-ion cell for hybrid electric vehicles. Journal of Power Sources, 161:628-639, 2006.

[14] M.W. Verbrugge and B.J. Koch. Electrochemical analysis of lithiated graphite anodes. J. Electrochem. Soc., 150, 2003.

[15] P. De Vidts, J. Delgado, and R.E. White. Mathematical modeling for the discharge of a metal hydride electrode. J. Electrochem. Soc., 142, 1995.

[16] C.Y. Wang, W.B. Gu, and B.Y. Liaw. Micro-macroscopic coupled modeling of batteries and fuel cells. part i: Model development. $J$. Electrochem. Soc., 145, 1998.

[17] C.Y. Wang, W.B. Gu, and B.Y. Liaw. Micro-macroscopic coupled modeling of batteries and fuel cells. part ii: Application to ni-cd and ni-mh cells. J. Electrochem. Soc., 145, 1998.

[18] J.W. Weidner and P. Timmerman. Effect of proton diffusion, electron conductivity, and charge-transfer resistance on nickel hydroxide discharge curves. J. Electrochem. Soc., 141, 1994. 